\title{
Análise da ocorrência de infecção hospitalar após cirurgia cardíaca em hospital de referência
}

\author{
Camila da Costa Nahum \\ Mestre em Saúde na Amazônia, do Núcleo de Medicina Tropical da Universidade Federal do Pará- UFPA \\ Marcelo Coelho Simões \\ Mestrando do Programa de Pós-graduação em Ciências Ambientais da Universidade do Estado do Pará- UEPA \\ $\triangle$ marcelo.uepa14@gmail.com \\ Andrea Cristina Beltrão Ferreira \\ Mestre em Doenças Infecciosas e Parasitárias da Fundação Hospital das Clínicas Gaspar Viana- HCGV
}

Andressa Ozela de Vilhena

Doutora em Biologia Parasitária da Amazônia da Universidade do Estado do Pará- UEPA

Cinthia de Oliveira Lisboa

Doutoranda em ortodontia da Universidade Federal Fluminense- UFF/RJ

Cléa Nazaré Carneiro Bichara

Doutora em Agentes Infecciosos e Parasitários da Universidade Federal do Pará- UFPA

\section{Resumo:}

O Ministério da Saúde conceitua infecção hospitalar como qualquer infecção que é adquirida após a admissão do paciente e se manifesta durante o período de internação ou após a alta, quando esta puder ser relacionada com a internação ou procedimentos hospitalares. Objetivou analisar o perfil dos pacientes submetidos à cirurgia cardíaca e a incidência de infecção hospitalar no pós-operatório. Trata-se de um estudo com abordagem quantitativa, descritivo, longitudinal e de caráter retrospectivo. A amostragem consistiu em 316 pacientes que foram submetidos à cirurgia cardíaca na Fundação Hospital de Clínicas Gaspar Viana, no período de 2013-2014 e que atenderam aos critérios de inclusão e exclusão. Os dados foram coletados dos prontuários através de formulário estruturado. A análise foi feita por meio de comparações utilizando o odds ratio, teste qui quadrado e posteriormente regressão logística. O estudo foi aprovado por Comitê de Ética em Pesquisa. Identificou-se que a maior parte dos pacientes era do sexo masculino, com idade inferior a 61 anos e de raça parda, da região metropolitana de Belém e casados. As principais comorbidades encontradas foram Diabetes Metilus, Hipertensão Arterial Sistêmica e tabagismo. A maior parte foi admitido por Infarto agudo do miocárdio, porém os pacientes com valvopatia tiveram maior associação com infecção. A partir de tais achados propôs-se um protocolo específico para ser utilizado desde a unidade de internação, passando pelo bloco cirúrgico até a unidade de terapia intensiva, pois se acredita que as medidas preventivas vão muito além daquelas já muitas vezes debatidas no ambiente de cuidados intensivos.

Palavras-chave: Comorbidades, Cirurgia cardíaca, Infecção hospitalar, Internação.

\section{Analysis of the occurrence of nosocomial infection after cardiac surgery in a referral hospital}

\section{Abstract:}

The Ministry of Health conceptualizes hospital infection as any infection that is acquired after the patient's admission and manifests itself during the hospitalization period or after discharge, when it 
can be related to hospitalization or hospital procedures. It aimed to analyze the profile of patients submitted to cardiac surgery and the incidence of postoperative hospital infection. It was a study with a quantitative, descriptive, longitudinal and retrospective approach. Sample consisted of 316 patients who underwent cardiac surgery at the Gaspar Viana Hospital Foundation, in the period 2013-2014 and who met the inclusion and exclusion criteria. The data were collected from the medical records through the form. The analysis was made through comparisons using the odds ratio, chi-square test and later logistic regression. The study was approved by the Research Ethics Committee. It was identified that the majority of the patients were male, less than 61 years old, from the metropolitan area of Belém and married. The main comorbidities were Metilus Diabetes, Systemic Arterial Hypertension and smoking. Most patients were admitted for Acute myocardial infarction, but patients with valvular disease had a higher association with infection. Conclusion: Based on these findings, a specific protocol was proposed to be used from the hospitalization unit, through the surgical unit to the intensive care unit, because it is believed that preventive measures go far beyond those already discussed in the intensive care setting.

Keywords: Comorbidities, Cardiac surgery, Hospital infection, Hospitalization.

\section{Análisis de la incidencia de infección nosocomial después de la cirugía cardíaca en el hospital de referencia}

\section{Resumen:}

El Ministerio de Salud conceptualiza la infección hospitalaria como cualquier infección que se adquiere con posterioridad al ingreso del paciente y se manifiesta durante el período de internación o posterior al alta, cuando puede estar relacionada con la hospitalización o procedimientos hospitalarios. Su objetivo analizar el perfil de los pacientes sometidos a cirugía cardiovascular y la prevalencia de la infección en el hospital después de la cirugía. Estudio con enfoque cuantitativo, descriptivo, longitudinal y de carácter retrospectivo. La muestra consistió en el 316 pacientes que se han sometido a una cirugía de corazón en la Fundación Hospital Gaspar Viana, en el periodo de 2013 a 2014, y que se han cumplido todos los criterios de inclusión y exclusión. La información se obtuvo de los registros a través del formulario de contacto. El análisis se ha realizado a través de las comparaciones mediante el odds-ratio, la prueba de la ji al cuadrado y, posteriormente, el método de regresión logística. El estudio fue aprobado por el Comité de Ética de la Investigación. Se identificó que la mayor parte de los pacientes eran de sexo masculino, con una edad inferior a los 61 años de edad y de la raza parda de la región metropolitana de Belém y el matrimonio. Las principales comorbilidades encontradas fueron la Diabetes de Metilus, hipertensión arterial sistémica, y el consumo de tabaco. La mayor parte ha sido aceptada por el infarto agudo de miocardio, sin embargo, en los pacientes con valvopatia han tenido una mayor asociación con la infección. Se ha propuesto un protocolo específico para ser utilizado desde la unidad de estancia en el hospital, pasando por el bloque quirúrgico, hasta que en la unidad de cuidados intensivos, ya que se considera que las medidas de prevención que van mucho más allá, ya que muchas veces se debate en un ambiente de cuidados intensivos.

Palabras clave: Comorbilidades, Cirugía cardíaca, Infección hospitalaria, Hospitalización.

\section{INTRODUÇÃO}

A portaria 2616/1998 do Ministério da Saúde conceitua infecção hospitalar como qualquer infecção que é adquirida após a admissão do paciente e se manifesta durante o período de internação ou após a alta, quando esta puder ser relacionada com a internação ou procedimentos hospitalares. Outro termo que vem sendo utilizado com frequência é Infecção 
Relacionada à Assistência à Saúde (IRAS), pois este abrange infecções adquiridas durante assistência ambulatorial, cuidados domiciliares, além de infecções ocupacionais adquiridas por profissionais de saúde. Este termo enfatiza o fato de grande parte das infecções em centros de saúde ser adquiridas durante procedimentos assistenciais, que podem ocorrer não só em hospitais, mas em clínicas, ambulatórios e unidades de saúde (TEIXEIRA et al., 2012).

Hospitais são lugares que oferecem um ambiente propício às bactérias devido aos procedimentos invasivos realizados, doença de base dos pacientes (baixa imunidade) e uso de terapia antimicrobiana. A implementação de terapias antimicrobianas e constantes mudanças geraram um aumento da resistência bacteriana, tornando muitas das drogas menos efetivas. Esse fato constitui um agravante para o aumento das taxas de infecção hospitalar, assim como de custos para o desenvolvimento de antibióticos mais efetivos (SANTANA et al., 2014).

No decorrer dos anos houve maior desenvolvimento da tecnologia em saúde e a realização de procedimentos invasivos no Brasil se tornou mais frequente, principalmente a partir dos anos 90. Como consequência da maior necessidade de haver uma sistematização e organização em torno das medidas a serem adotadas na prevenção e redução de índices de infecção, foi criado o Programa de Controle de Infecção Hospitalar (PCIH), delineado pela Lei no 9431 de janeiro de 1997 que dispõe da obrigatoriedade dos hospitais em estabelecerem a Comissão de Controle de Infecção Hospitalar (CCIH) para manter um programa de controle de infecção no hospital (BRASIL, 1997).

A cirurgia cardíaca é um procedimento que acarreta diversas alterações na homeostase orgânica e exige um cuidado intensivo. Devido a isso, o surgimento de complicações infecciosas não é uma realidade difícil de ocorrer devido ao trauma cirúrgico e aos fatores de risco que o paciente cardiopata em pós-operatório possui, como diabetes, realização de múltiplos procedimentos invasivos, como acesso venoso central, cateter vesical e ventilação mecânica; cirurgias prolongadas e hipotermia. Algumas dessas complicações também estão relacionadas a técnicas cirúrgicas, como a Circulação Extracorpórea (CEC), e intervenções realizadas devido complicações após a cirurgia, como a necessidade de diálise, e dentre tais complicações estão às infecções de sítio cirúrgico, pneumonias e infecções relacionadas a cateterismo central (SAHU et al., 2016). 
Existem alguns fatores relacionados ao paciente que são considerados preditores de infecções no período pós-operatório cardíaco, devido às consequências na homeostase orgânica do paciente, tais como: índice de massa corpórea $\geq 40 \mathrm{~kg} / \mathrm{m} 2$, hemodiálise no préoperatório, choque cardiogênico pré-operatório, idade $\geq 85$ anos, tratamento com imunossupressores no pré-operatório, diabetes melitus, tempo de CEC $\geq 200$ minutos, utilização de balão intra-aórtico e 3 ou mais vasos revascularizados (OLIVEIRA et al., 2010).

A identificação de tais fatores de risco e achados clínicos devem ser avaliados no préoperatório de pacientes que são atendidos em instituições que realizam cirurgias cardíacas e a análise de sua relação com desfechos após a cirurgia. Nesse contexto, protocolos e escores de risco tem sido elaborados e aplicados em diferentes instituições, com o objetivo de reduzir infecções após as cirurgias (LEDUR et al., 2011).

Dessa forma, esta pesquisa visa trazer contribuição para a área científica, trazendo novos achados que possibilitem a reflexão sobre fragilidades e potencialidades das medidas de cuidado e prevenção e consequentemente ajudem no direcionamento das práticas de saúde dos profissionais, com a finalidade de garantir uma assistência de qualidade ao paciente. Com base nisso, este estudo objetivou analisar o perfil dos pacientes submetidos à cirurgia cardíaca e a incidência de infecção hospitalar no pós-operatório em um hospital de referência do estado do Pará.

\section{METODOLOGIA}

Trata-se de um estudo com abordagem quantitativa, descritivo, longitudinal e de caráter retrospectivo. A pesquisa quantitativa busca traduzir em números as informações, para depois quantificá-las e analisá-las. Para isso requer o uso de técnicas estatísticas. Este tipo de pesquisa utiliza procedimentos estruturados e instrumentos formais para a coleta de dados e enfatiza a objetividade presente nos dados numéricos (GERHARDT; SILVEIRA, 2009; PRODANOV, 2013).

O estudo foi realizado na Fundação Hospital de Clínicas Gaspar Viana (FHCGV), que fornece um ótimo campo para pesquisa na área de cardiologia, por ser uma referência nessa 
área para o estado do Pará. Também é referência em nefrologia e psiquiatria. Realiza anualmente cerca de 500 cirurgias cardíacas dos diferentes tipos (incluindo adultos e crianças), dispondo de estrutura física, suporte técnico e profissional capacitado para tal tipo de tratamento.

A pesquisa foi desenvolvida dentro do SAME (Serviço de Arquivo Médico e Estatístico), local onde são armazenados os prontuários dos pacientes, por se tratar de um estudo retrospectivo com análise de prontuários.

O estudo foi submetido e sido aprovado à Plataforma Brasil para avaliação pelo Comitê de Ética em Pesquisa da Fundação Hospital de Clínicas Gaspar Viana, de acordo com o observado na portaria 466/12 que estabelece critérios para o estudo em seres humanos, sobre o protocolo 56725716.6.0000.0016. Por se tratar de uma pesquisa com dados secundários que foram coletados de prontuários armazenados no hospital, dispensou o uso de Termo de Consentimento Livre e Esclarecido. Ressalta-se que identidade dos pacientes foi mantida sob sigilo absoluto, assegurando-se a privacidade quanto aos dados confidenciais envolvidos na pesquisa. A identificação foi feita através de numeração em ordem crescente, e não houve qualquer ônus econômico-financeiro para as Instituições envolvidas nesta.

Além disso, foi preenchido o Termo de Compromisso para Utilização de Dados e/ou Prontuários dos Pacientes, conforme modelo do Serviço de Graduação e Pós-graduação e Pesquisa (SEGRAP), onde foram preservados todos e quaisquer dados obtidos.

\section{A amostra}

A amostra do estudo consistiu de pacientes que foram submetidos à cirurgia cardíaca na FHCGV no período de 2013-2014 e que atenderam aos critérios de inclusão e exclusão. Esse período foi escolhido, pois, acredita-se que garantirá uma dimensão populacional adequada para a pesquisa. Ao final da pesquisa chegou-se ao total de 316 prontuários pesquisados.

\section{Critérios de inclusão e exclusão}

Foram incluídos na pesquisa os pacientes maiores de 18 anos, de ambos os sexos, que foram submetidos à cirurgia cardíaca com esternotomia mediana longitudinal e após isso 
foram internados em ambiente de cuidados intensivos. A pesquisa envolveu somente cirurgias cardíacas em adultos devido à cirurgia pediátrica e neonatal envolver aspectos, como tipo da cardiopatia, uso de antibióticos e perfil de infecção diferentes dos adultos.

Foram excluídos da pesquisa os prontuários que não foram devidamente identificados, localizados e que estavam incompletos, daqueles pacientes que evoluíram a óbito ainda na sala de cirurgia ou 24 horas após a cirurgia, pois nesse período não é possível identificar infecções pós-operatórias. Além disso, foram excluídas as cirurgias que não precisaram de internação em ambiente de cuidados intensivos, como implante de marca-passo e drenagem de pericárdio.

\section{Obtenção de dados}

A coleta de dados foi feita nos prontuários do pacientes internados no período de 20132014, que ficaram armazenados no SAME. Foi utilizado um formulário para ajudar a direcionar a busca por informações.

O instrumento de coleta de dados apresenta a seguinte divisão que contem as variáveis do estudo:

- Dados sócio demográficos: sexo, idade, raça, escolaridade, naturalidade, estado civil, procedência.

-Antecedentes pessoais e comorbidades: Diabetes Melitus (DM), Hipertensão Arterial Sistêmica (HAS), tabagismo, Doença Pulmonar Obstrutiva Crônica (DPOC), etilismo, Insuficiência Renal Crônica (IRC).

-Evolução durante internação (fatores que podem interferir na susceptibilidade a infecções no pós-operatório): causa da internação, ocorrência de infecções durante a internação pré-operatória e sua topografia, agente e qual antibiótico utilizado para tratar. Uso de antibiótico profilático, banho com clorexidina, tricotomia, tempo de internação préoperatório total.

-Durante a cirurgia (variáveis relacionadas ao processo cirúrgico que podem ser relacionadas à susceptibilidade às infecções). 
-Após a cirurgia (variáveis relacionadas ao pós-operatório que podem ser relacionadas com a susceptibilidade a infecções).

\section{Análise estatística}

Para avaliar os fatores de risco para infecção hospitalar foram aplicados métodos estatísticos descritivos e inferenciais. Para descrever os grupos de pacientes que contraíram Infecção (Caso $n=129$ ) e os isentos de infecção hospitalar (Controle $n=187$ ). Nas variáveis quantitativas foi aplicado o cálculo do Ponto de corte e posteriormente transformadas em variáveis qualitativas dicotômicas. As variáveis qualitativas foram apresentadas por distribuições de frequências absolutas e relativas. A comparação entre os grupos foi realizada pelo Odds Ratio seguido do teste do qui-quadrado. Foi previamente definido que as variáveis que obtivessem p-valor $<0.05$ seriam submetidas ao modelo multivariado de regressão logística (AYRES et al., 2007). Foi previamente fixado o nível de significância alfa $=0.05$ para rejeição da hipótese de nulidade. O processamento estatístico foi realizado nos softwares SAM (Statistical Analysis Model) versão 1.0 e BioEstat versão 5.3.

\section{RESULTADOS}

Quanto ao perfil dos pacientes estudados, submetidos à cirurgia cardíaca, estão representados na Tabela 01.

Tabela 1 - Caracterização de pacientes admitidos na UCA/FHCGV.

\begin{tabular}{lll}
\hline Caracterização & N & \% \\
\hline Sexo & & \\
Masculino & 216 & 68,3 \\
Feminino & 100 & 31,6 \\
Idade & & \\
$<61$ a & 171 & 54.1 \\
Raça & & \\
Branca & 101 & 31,7 \\
Negra & 39 & 12.3 \\
Parda & 176 & 55,6
\end{tabular}




\begin{tabular}{lll} 
Escolaridade & & \\
Analfabeto & 45 & 14.2 \\
Fundamental & 230 & 72.7 \\
Médio & 32 & 10.1 \\
Superior & 9 & 0.3 \\
Origem & & \\
Interior & 130 & 41.1 \\
RM Belém & 186 & 58.8 \\
Estado Civil & & \\
Solteiro & 56 & 17.7 \\
Casado & 166 & 52,5 \\
Viúvo & 22 & 6.9 \\
Divorciado & 12 & 3.7 \\
U Estável & 60 & 18.9 \\
\hline
\end{tabular}

Fonte: dados do estudo, 2016.

Entre as comorbidades, a HAS apresentou índices mais elevados, seguido do tabagismo e diabetes melitus (Tabela 2).

Tabela 2 - Cirurgia prévia e comorbidades apresentadas em pacientes admitidos na UCA/FHCGV

\begin{tabular}{lll}
\hline Histórico & $\mathbf{n}$ & $\mathbf{\%}$ \\
\hline Cirurgia anterior & 5 & \\
Revasc. Miocárdio & 20 & 1.5 \\
Troca válvula & 18 & 6.3 \\
Angioplastia & & 5.6 \\
\hline Comorbid. Ant & 114 & \\
DM & 223 & 36,0 \\
HAS & 150 & 70.5 \\
Tabagismo & 5 & 47.4 \\
DPOC & 84 & 1.5 \\
Etilismo & 15 & 26.5 \\
Renal Crônico & 4.7
\end{tabular}

Fonte: Dados do estudo, 2016. *UCA- Unidade Coronariana. **FHCGV- Fundação Hospital de Clínicas Gaspar Viana. ${ }^{* * *}$ DM- Diabetes Melitus. ${ }^{* * * *}$ HAS- Hipertensão Arterial Sistêmica. ${ }^{* * * * *}$ DPOCDoença Pulmonar Obstrutiva Crônica. 
As maiores partes dos pacientes foram internados devido IAM (52.8\%), seguido de valvulopatia $(17,72 \%)$ (Tabela 3$)$.

Tabela 3 - Quanto ao desenvolvimento de infecção no pós-operatório.

\begin{tabular}{lcc}
\hline & N & \% \\
\hline Desenvolveu infecção & 129 & 40.8 \\
Sem infecção & 187 & 59.1 \\
Total & 316 & 100 \\
\hline
\end{tabular}

Fonte: Dados do estudo, 2016.

Esta informação está de acordo com outros estudos sobre cirurgias cardíacas que evidenciaram a insuficiência coronariana como o diagnóstico médico mais frequente (OLIVEIRA; FANTINATI, 2013).

Tabela 4 - Evolução durante a internação pré-operatória de pacientes admitidos na FHCGV, conforme a presença de Infecção $(n=129)$ e Sem Infecção (n=187).

\begin{tabular}{|c|c|c|c|c|c|c|c|}
\hline \multirow[b]{2}{*}{ Evolução Internação } & \multicolumn{2}{|c|}{ Infecção } & \multicolumn{2}{|c|}{ S/infecção } & \multicolumn{3}{|c|}{ odds } \\
\hline & $\mathbf{n}$ & $\%$ & $\mathbf{n}$ & $\%$ & p-valor & Ratio & IC $95 \%$ \\
\hline Causa & & & & & $0.0131^{*}$ & 1.92 & 1.07 a 3.45 \\
\hline IAM & 60 & 46.5 & 107 & 57.2 & & & \\
\hline Valvulopatia* & 31 & 24.0 & 25 & 13.4 & & & \\
\hline ICC & 21 & 16.3 & 14 & 7.5 & & & \\
\hline Angina instável & 23 & 17.8 & 42 & 22.5 & & & \\
\hline Aneurisma Aorta & 4 & 3.1 & 4 & 2.1 & & & \\
\hline Tratou a infecção anterior & & & & & 0.1709 & 1.72 & 0.86 a 3.46 \\
\hline Sim, tratou & 19 & 14.7 & 17 & 9.1 & & & \\
\hline Não tratou & 110 & 85.3 & 170 & 90.9 & & & \\
\hline \multicolumn{8}{|l|}{$\begin{array}{l}\text { Topografia infecção } \\
\text { anterior }\end{array}$} \\
\hline Pneumonia & 17 & 13.2 & 14 & 7.5 & 0.1390 (ns) & 1.87 & 0.88 a 3.95 \\
\hline Infec T Urinário & 5 & 3.9 & 3 & 1.6 & 0.3685 (ns) & 2.47 & 0.58 a 10.5 \\
\hline Endocardite & 3 & 2.3 & 5 & 2.7 & 0.8645 (ns) & 0.86 & 0.20 a 3.69 \\
\hline Corrente Sanguínea & 0 & 0.0 & 1 & 0.5 & $\mathrm{n} / \mathrm{a}$ & --- & --- \\
\hline T Internação > 26 dias & 66 & 51.2 & 90 & .1 & 0.6775 & 1.12 & 0.72 a 1.78 \\
\hline \multicolumn{8}{|l|}{ Cuidados pré-operatórios } \\
\hline Banho com clorexidina & 87 & 67.4 & 117 & 62.6 & 0.4408 (ns) & 1.23 & 0.77 a 1.98 \\
\hline Tricotomia & 88 & 68.2 & 117 & 62.6 & 0.3606 (ns) & 1.23 & 0.79 a 3.06 \\
\hline
\end{tabular}


A maior parte dos pacientes passou por cirurgia cardíaca de caráter eletivo nos dois grupos (87.9\%), sendo que não houve correlação com maiores índices de infecção. De modo geral, as cirurgias mais urgentes são as que apresentam lesões graves como lesão de tronco de coronária esquerda, no caso de IAM, ou insuficiência cardíaca no qual a fração de ejeção do Ventrículo esquerdo encontra-se muito comprometida (Tabela 5).

Tabela 5 - Eventos durante a cirurgia em pacientes admitidos na CTI/HCGV.

\begin{tabular}{|c|c|c|c|c|c|c|c|}
\hline \multirow[b]{2}{*}{ Durante a cirurgia } & \multicolumn{2}{|c|}{ Infecção } & \multicolumn{2}{|c|}{$\begin{array}{c}\text { S/ } \\
\text { infecção }\end{array}$} & \multirow[b]{2}{*}{ p-valor } & \multicolumn{2}{|l|}{ odds } \\
\hline & $\mathbf{n}$ & $\%$ & $\mathbf{n}$ & $\%$ & & Ratio & IC 95\% \\
\hline Opção pela Cirurgia & & & & & $0.4844(\mathrm{~ns})$ & 0.73 & 0.37 a 1.45 \\
\hline Eletiva & 111 & 86.0 & 167 & 89.3 & & & \\
\hline Urgência & 15 & 11.6 & 19 & 10.2 & & & \\
\hline Cirurgia realizada & & & & & $0.0013^{*}$ & 3.86 & 1.81 a 8.13 \\
\hline Revasc Miocardio & 79 & 61.2 & 144 & 77.0 & & & \\
\hline Troca Válvula Aórtica & 23 & 17.8 & 22 & 11.8 & & & \\
\hline Troca Válvula Mitral* & 25 & 19.4 & 11 & 5.9 & & & \\
\hline Corr Aneur Aorta & 3 & 2.3 & 2 & 1.1 & & & \\
\hline Corr CIV CIA & 1 & 0.8 & 2 & 1.1 & & & \\
\hline Uso de CEC & & & & & 0.9402 & 1.08 & 0.41 a 2.89 \\
\hline Usou CEC & 122 & 94.6 & 176 & 94.1 & & & \\
\hline Repique Antib após CEC & 111 & 86.0 & 153 & 81.8 & & & \\
\hline
\end{tabular}

Os antibióticos utilizados foram cefepime e cefuroxima, sendo este último mais utilizado em ambos os grupos. Não houve maior exposição à infecção em nenhum dos grupos. Esses antibióticos são iniciados na sala de cirurgia e continuam sendo utilizados nos dias seguintes no pós-operatório (Tabela 6).

Tabela 6 - Eventos pós-cirúrgicos em pacientes admitidos na UCA/FHCGV, conforme a presença de Infecção ( $n=129)$ e Sem infecção $(n=187)$.

\begin{tabular}{|c|c|c|c|c|c|c|c|}
\hline \multirow[b]{2}{*}{ Após a cirurgia } & \multicolumn{2}{|c|}{ Infecção } & \multicolumn{2}{|c|}{ Sem infecção } & \multicolumn{3}{|c|}{ Odds } \\
\hline & $\mathbf{N}$ & $\%$ & $\mathbf{n}$ & $\%$ & $\mathrm{p}$-valor & Ratio & IC $95 \%$ \\
\hline \multicolumn{8}{|l|}{$\begin{array}{l}\text { Antibiótico } \\
\text { profilático }\end{array}$} \\
\hline Prof Cefepime & 28 & 21.7 & 43 & 23.0 & $0.9284(\mathrm{~ns})$ & 0.15 & 0.54 a 1.59 \\
\hline Prof Cefuroxima & 86 & 66.7 & 121 & 64.7 & 0.8103 (ns) & 1.09 & 0.67 a 1.75 \\
\hline \multicolumn{8}{|l|}{ Complicações PO } \\
\hline Reintubação & 11 & 8.5 & 2 & 1.1 & $0.0028^{*}$ & 1.89 & 1.88 a 39.59 \\
\hline
\end{tabular}




\begin{tabular}{lccccccc} 
Fibrilação atrial & 29 & 22.5 & 23 & 12.3 & $0.0248^{*}$ & 2.06 & 1.13 a 3.77 \\
Fibr Vent Taquic & 13 & 10.1 & 13 & 7.0 & 0.4321 & 1.5 & 0.67 a 3.51 \\
Taquic Suprav & 3 & 2.3 & 2 & 1.1 & 0.6739 & 2.2 & 0.36 a 13.3 \\
Sangramento & 4 & 3.1 & 5 & 2.7 & 0.9047 & 1.16 & 0.36 a 4.42 \\
Insuf Resp & 15 & 11.6 & 2 & 1.1 & $0.0001^{*}$ & 12.17 & 2.73 a 54.21 \\
AVC & 6 & 4.7 & 2 & 1.1 & 0.1036 & 4.51 & 0.89 a 22.7 \\
Insuf Renal & 19 & 14.7 & 7 & 3.7 & $0.0010^{*}$ & 4.44 & 1.80 a 10.90 \\
Derrame Pleural & 10 & 7.8 & 5 & 2.7 & 0.0691 & 3.05 & 1.02 a 9.17 \\
\hline
\end{tabular}

Fonte: dados do estudo, 2016. *AVE- Acidente Vascular Encefálico.

\section{DISCUSSÃO}

$\mathrm{Na}$ pesquisa foi identificada uma predominância de pacientes do sexo masculino submetidos à cirurgia cardíaca, sendo essa uma realidade comum, geralmente identificada na rotina de cuidados ao paciente cardiopata e em diversos estudos.

Quanto à escolaridade a maior parte dos pacientes tinha o ensino fundamental 230 indivíduos (72.7\%), seguido de analfabetismo 45 (14,2\%). Ocorre uma predominância de paciente de classe mais humilde, onde muitos não tiveram o acesso à educação, ou por circunstâncias adversas não podem dar continuidade aos estudos.

Quanto à raça dos pacientes submetidos à cirurgia cardíaca, a maior parte se autodenominou parda 176 indivíduos (55,6\%), seguida de branco e negro 101 (31,7\%) e 39 (12.3\%), respectivamente. A raça parda é mais comumente presente na região norte, o que poderia justificar seu maior predomínio entre aqueles que realizaram cirúrgica cardíaca.

No estudo também foi identificado uma predominância de pacientes casados 166 (52,5\%) e em união estável 60 (18.9), respectivamente, quando comparado aos outros grupos, sendo que esta realidade pode estar relacionada à maior idade entre tais pacientes, que consequentemente está exposta a maiores fatores de risco.

A principal razão para tal achado é que o sexo masculino tem sido identificado como o gênero mais exposto a fatores de risco para diversas doenças, entre elas, as Doenças Crônicas Degenerativas, que incluem as doenças cardiovasculares. Em um estudo transversal com dados de 2011 do Sistema de Vigilância de Fatores de Risco e Proteção para doenças crônicas por inquérito telefônico, que incluiu adultos residentes nas capitais brasileiras, os 
homens apresentaram prevalência da maior parte dos fatores de risco, como tabagismo, consumo de bebida alcóolica, excesso de peso e inatividade física. É conhecido que esses fatores predispõem a doenças cardiovasculares, e como os homens são mais expostos aos mesmos, tendem a apresentar com maior frequência tais doenças (MALTA et al., 2011).

Um achado muito interessante no estudo e que reflete uma tendência observada na atualidade foi verificar que cerca de $54 \%$ dos pacientes estudados estavam em uma faixa etária menor que 61 anos, ou seja, são pacientes cada vez mais jovens acometidos por doenças cardiovasculares. Isso se deve em grande parte à transição epidemiológica pelo qual o Brasil tem passado e às mudanças de hábitos de vida. Em 2011, quase a metade dos adultos ( $\geq 18$ anos de idade) em capitais brasileiras relataram excesso de peso (48,5\%), 17,0\% referiram consumo abusivo de álcool, 20,0\% consumiam frutas e hortaliças em quantidade insuficiente e 14,0\% eram inativos fisicamente. Essas mudanças refletem no desenvolvimento precoce de doenças cardiovasculares (DUARTE; BARRETO, 2012).

Estudos mostram que pacientes com menor nível de escolaridade apresentam maior tendência a apresentar fatores de risco para doenças cardiovasculares, quando comparados a pacientes com nível superior. Quando compreendemos que a pouca escolaridade pode dificultar a aquisição de conhecimento, das orientações terapêuticas no cuidado com a doença, e na aquisição de novos hábitos de vida, podemos perceber a necessidade da utilização de métodos de educação adequados para que os conhecimentos preventivos alcancem a maior parte da população. Além disso, meios que possibilitem uma alimentação saudável e prática de atividade física são mais limitados entre a classe mais humilde (MENDES et al., 2011).

Observamos um maior número de pacientes admitidos da própria capital e região metropolitana 186 pacientes (58.8\%), mas também uma quantidade expressiva de pacientes provenientes do interior do estado 130 (41.1\%). No nosso Estado a FHCGV é a referência em assistência cardiovascular, agregando pacientes que vem de diferentes locais do Estado e não somente da região metropolitana, em busca de tratamento especializado. Sendo o Pará um estado de amplas dimensões geográficas, é importante ressaltar as dificuldades encontradas por pacientes provenientes do interior, com dificuldades para adquirir transporte e vias de acesso. 
Observa-se um maior índice de necessidade de nova cirurgia entre os pacientes submetidos à troca de válvula aórtica ou mitral, sendo este fato relacionado à característica do próprio procedimento, pois a válvula precisa ser trocada depois de certo tempo, apresentado desgaste.

A (HAS) é apontada como fator de risco para complicações e doenças cardiovasculares na sociedade atual, mas também é associada a outras doenças como edema agudo de pulmão, insuficiência renal e acidente vascular encefálico (AVE), explicando 54\% das mortes por acidente vascular encefálico e $47 \%$ daquelas por doença isquêmica do coração. Esta doença é silenciosa e inicialmente podem não ocorrer sintomas, o que por vezes pode levar a uma não adesão adequada do tratamento. Esta negligência acarreta em um incorreto controle dos níveis pressóricos levando às lesões em órgãos- alvo (coração, rins, cérebro). Portanto, a orientação quanto aos males causados e incentivo ao tratamento é de suma importância e tarefa de todo profissional de saúde (SANTOS; MOREIRA; MAIA, 2013).

Além disso, os idosos possuem o hábito de fumar, consumir álcool e alimentos hipercalóricos, associado ao sedentarismo, os quais são considerados fatores de risco para IAM, o que mostra falhas em ações efetivas voltadas para o seu controle e prevenção. Esses fatores associados são muito danosos ao paciente o expondo não somente a doenças cardiovasculares, mas também a danos cerebrais, pulmonares e renais. Medidas preventivas é a chave, porém é necessário maior investimento em programas como o Hiperdia da Atenção Básica (PINHEIRO et al., 2013).

Dos 316 pacientes estudados, 40,8\% desenvolveram algum tipo de infecção no pósoperatório de cirurgia cardíaca. Essa quantidade expressiva de casos de infecção em pósoperatório de cirurgia cardíaca ocorre devido ser um procedimento extremamente invasivo, realizado geralmente em pacientes com comorbidades que o predispõe à infecção. As complicações infecciosas após cirurgias cardíacas limpas ocorrem em até 3,5\% dos pacientes, e contribuem de forma relevante para a elevada morbidade e mortalidade dos pacientes (SOARES et al., 2011).

A partir da divisão do grupo que desenvolveu algum tipo de infecção e o grupo sem infecção foram feitas correlações com as diferentes variáveis a que ele foi exposto. 
A maior parte dos pacientes foram internados devido IAM (167-52.8\%), seguido de valvulopatia (56-17,72\%). Esta informação está de acordo com outros estudos sobre cirúrgicas cardíacas que evidenciaram a insuficiência coronariana como o diagnóstico médico mais frequente (OLIVEIRA; FANTINATI, 2013).

Este dado também corrobora a estimativa nacional de que o IAM é a primeira causa de morte em nosso país. O Departamento de informática do SUS tem registrado cerca de 100.000 óbitos anuais devido à doença (DATASUS, 2014).

Entretanto, ao se estabelecer o comparativo percebe-se que entre os pacientes que apresentavam valvulopatia o valor de $\mathrm{p}$ foi significativo, indicando que tal doença é tida como fator de risco para o desenvolvimento de infecções e pacientes com valvulopatia tem 1,95 de chances a mais de desenvolver infecção. Esta realidade está relacionada ao fato de que pacientes com tal acometimento de válvulas geralmente são pacientes que já passaram por trocas de válvula anteriores, são mais debilitados e até mesmo o tempo de CEC de cirurgia de troca de válvula é maior, fatores que o predispõem a maiores complicações e infecções. No estudo de Torrati e Dantas (2012), os pacientes submetidos à troca de válvula tiveram tempo de CEC superior ao de outras cirurgias.

Com relação ao tratamento de infecção antes do procedimento cirúrgico, temos que nos dois grupos foram desenvolvidas infecções que precisaram ser tratadas, sendo a maior parte das infecções pneumonia e infecção do trato urinário. $O$ fato de o paciente ter apresentado infecção anterior não apresentou relação significativa com o desenvolvimento de infecção no pós-operatório, demonstrando que ocorrendo um tratamento adequado com uso racional de antibióticos existe segurança em se realizar o procedimento cirúrgico sem o desenvolvimento de novas infecções.

A média de tempo de internação pré-operatório encontrada foi de 26 dias de internação para os dois grupos. 0 tempo de internação neste estudo não foi associado a maiores níveis de infecção no pós-operatório, porém o tempo em questão é considerado alto, levando em conta que o paciente é exposto ao ambiente hospitalar e sua microbiota por longo período, o que pode levar ao surgimento de infecções antes mesmo de ocorrer à cirurgia, como foi constatado anteriormente, onde muitos desenvolveram pneumonia e infecção do trato urinário (IZAIAS et al., 2014). 
A maior parte dos pacientes realizou banho com clorexidina e tricotomia préoperatória, no entanto não houve relação com aumento de infecção entre aqueles que não realizaram.

É importante atentar para a forma que a tricotomia é realizada. Se houverem cortes microscópicos na pele o risco de infecção pode ser aumentado devido os mesmos servirem de foco para invasão bacteriana. Quanto ao banho pré-operatório, o produto indicado é a clorexidina $2 \%$ degermante duas horas antes do procedimento. Em um estudo de revisão sistemática incluindo sete ensaios envolvendo 10.157 participantes mostraram não haver variações significativas no número de infecção em pacientes que realizaram banho préoperatório com clorexidina ou com outro produto como sabão neutro (WEBSTER; OSBORNE, 2015).

A maior parte dos pacientes passou por cirurgia cardíaca de caráter eletivo nos dois grupos (87.9\%), sendo que não houve correlação com maiores índices de infecção. De modo geral as cirurgias mais urgentes são as que apresentam lesões graves como lesão de tronco de coronária esquerda, no caso de IAM, ou IC no qual a fração de ejeção do Ventrículo esquerdo encontra-se muito comprometida. Índices semelhantes são apresentados em outros estudos, como no Instituto Dante Pazzanese de Cardiologia, em São Paulo, 83,2\% das cirurgias de revascularização ocorreram eletivamente, 10,9\% em caráter de urgência e 5,9\% de emergência (TONIAL; MOREIRA, 2011).

A cirurgia realizada com maior frequência foi à cirurgia de revascularização do miocárdio, nos dois grupos, com uma porcentagem de 70.6\% das cirurgias. Foi seguida pela cirurgia de válvula aórtica/mitral, com 25.6\%. Esses números são compatíveis com a realidade atual, que mostram as doenças coronarianas como as mais prevalentes, que geram mais óbitos no país e consequentemente necessidade de intervenção cirúrgica (KAUFFMAN et al., 2011).

No entanto ao se estabelecer a correlação entre os grupos, foi evidenciado que os pacientes que realizaram cirurgias de troca de válvula mitral apresentaram chance aumentada de apresentar algum tipo de infecção no pós-operatório. Esse achado está em harmonia com o índice anterior, que mostrou uma correlação entre pacientes internados por valvulopatia e chances aumentadas de infecção. As causas para tal característica pode ser o fato de que nas cirurgias de troca de válvula por vezes exigem maior tempo de CEC, maior 
tempo de internação e os pacientes estão mais debilitados, como mencionado anteriormente. Porém verificou-se a ausência de outros estudos anteriores que evidenciassem um maior índice de infecção entre este tipo de cirurgia (ROCHA, 2013).

A maior parte dos pacientes foi submetida à cirurgia com uso de CEC, porém nos dois grupos não houve maior associação com ocorrência de infecção. Sabe-se que o uso de CEC induz a resposta inflamatória sistêmica por meio da ativação do sistema complemento, sendo que isso ocorre principalmente devido ao contato do sangue com superfícies diferentes dos vasos sanguíneos, que são os tubos utilizados. Isso leva a uma liberação de mediadores inflamatórios que acarretam em uma resposta inflamatória sistêmica e depois a uma imunodeficiência temporária. Por isso uma monitoração rigorosa dos pacientes no pósoperatório é necessária como medida para avaliar também os efeitos da CEC (LIGUORI; KANAS; MOREIRA, 2014).

Nos dois grupos a maior parte dos pacientes realizou antibiótico após a CEC. A antibioticoprofilaxia cirúrgica é considerada uma medida fundamental para prevenção de infecções, especialmente em cirurgias prolongadas, e, no caso da cirurgia cardíaca, devido à CEC. Essa prática reduz eventos adversos e infecções. Um estudo em um hospital de São Paulo revelou resultados semelhantes, tendo bons índices de adesão à dose na cirurgia cardíaca (SCHMITT; LACERDA, 2015).

Os antibióticos utilizados foram cefepime e cefuroxima, sendo este último mais utilizado em ambos os grupos. Não houve maior exposição à infecção em nenhum dos grupos. Esses antibióticos são iniciados na sala de cirurgia e continuam sendo utilizados nos dias seguintes no pós-operatório. As recomendações internacionais são que uma cefalosporina deva ser administrada na sala de cirurgia e deve continuar de 24-72 horas. Em geral são utilizadas cefazolina ou cefuroxima, sendo que de modo geral as cefalosporinas são eficazes na redução de infecções, especialmente de sítio cirúrgico, não sendo identificada superioridade de uma sobre a outra. Em nosso estudo foi identificada a utilização de cefepime, sendo que em alguns prontuários a justificativa para sua utilização foi à falta de disponibilidades dos outros antibióticos na instituição.

A tabela também mostra as principais complicações apresentadas no pós-operatório, sendo que a reintubação, fribrilação atrial, insuficiência respiratória e insuficiência renal se demonstraram como fatores que aumentaram as chances de o paciente desenvolver infecção, 
com maior associação $(\mathrm{p}<0,05)$. De modo geral, as complicações que os pacientes apresentam tendem a prolongar seu tempo de internação na unidade intensiva, o que os expõem por maior tempo a fatores de risco conhecidos que são característicos desse ambiente, como os procedimentos invasivos necessários.

A reintubação é a necessidade de o paciente ser novamente entubado por ter ocorrido alguma descompensação hemodinâmica que torna necessário o uso do ventilador mecânico. Em uma coorte histórica realizada num hospital público de Uberaba, Minas Gerais, com 384 pacientes, a necessidade de reintubação apresentou forte associação e elevado fator de risco para desenvolvimento de Infecção de Sítio Cirúrgico (ISC). As causas para que a reintubação esteja associada a infecções pós-operatórias ainda carecem de maiores estudos, porém o fato de esta prática estar associada à maior gravidade dos pacientes, que tem seus mecanismos de defesa diminuídos e necessidade de novas intervenções invasivas pode ser um fato que contribui para sua ocorrência (SILVA; BARBOSA, 2012).

A fibrilação atrial é uma das arritmias mais comuns no pós-operatório de cirurgia cardíaca, ocorrendo em cerca de 30\% dos pacientes submetidos à RVM e em $40 \%$ de cirurgia valvular. Existem alguns fatores de risco para o desenvolvimento da arritmia, como: idade avançada, HAS, ventilação mecânica prolongada, tempo prolongado de CEC, cirurgia prévia. O tempo de permanência na UTI dos pacientes que desenvolvem FA aumenta em média dois a quatro dias em relação aos que mantém o ritmo sinusal, o que gera aumento de custos, porém não ocorre aumento de mortalidade dos pacientes. Essa pode ser a razão para a mesma estar associada ao desenvolvimento de infecções no pós-operatório (SOBRAL et al., 2013).

A insuficiência respiratória é uma complicação que está relacionada com a necessidade de oxigenoterapia, ventilação mecânica ou reintubação. Todas essas variáveis, como já mencionados, estão associadas à maior gravidade do paciente e ao prolongamento da internação. A intubação endotraqueal é uma medida utilizada que está relacionada à infecção, como pneumonia, e devido a isso, a associação entre pacientes com insuficiência respiratória e desenvolvimento de infecção esteja presente. As complicações pulmonares constituem a segunda causa mais frequente de morbidade e mortalidade no período pós-operatório de cirurgia cardíaca. Muitos são os fatores que podem contribuir para desencadear tais alterações, sendo alguns deles o uso de circulação extracorpórea, que ocasiona discretas 
alterações metabólicas e eletrolíticas no paciente, o grau de manipulação cirúrgica, o número de drenos pleurais, entre outros (BECCARIA et al., 2015).

Os pacientes que desenvolveram IRA também tiveram maiores chances de desenvolver infecções. Em um estudo de corte transversal com 321 pacientes em São Paulo, no Instituto Dante Pazzanese a idade, o uso de insulina, tempo de CEC, baixo débito cardíaco e cirurgia valvar estiveram associados ao risco de desenvolvimento de IRA. Além disso, durante o acompanhamento de tais pacientes 11 morreram por septicemia e choque cardiogênico, 6 precisaram de hemodiálise e 75 tiveram sua internação prolongada. Esses dados mostram que pacientes acometidos por IRA tem como consequência a exposição a mais procedimentos invasivos (uso de cateter para diálise, a própria hemodiálise), que acabam aumentando seu tempo de internação e acarretando em maior exposição a patógenos (SAMPAIO et al., 2013).

Em um estudo com 14 casos controle onde se analisou o comprometimento renal foi concluído que os fatores pós-operatórios que foram associados à lesão renal aguda foram infecção, cirurgias anteriores, cirurgias de emergência, insuficiência cardíaca congestiva e baixo débito cardíaco. Os fatores associados podem estar relacionados à instabilidade hemodinâmica resultante e ao trauma cirúrgico (YI et al., 2016).

\section{CONSIDERAÇÕES FINAIS}

Neste estudo, identificou-se que a maior parte dos pacientes era do sexo masculino, com idade inferior a 61anos, de raça parda, sendo analfabetos ou com ensino fundamental, da região metropolitana de Belém e casados. As principais comorbidades encontradas foram DM, HAS e tabagismo.

Um número considerável dos pacientes do estudo desenvolveu infecção. A maior parte foi admitido por IAM, porém os pacientes com valvopatia tiveram maior associação com infecção. A principal infecção desenvolvida antes da cirurgia foi à pneumonia. $O$ tempo de internação pré-operatório foi maior que 26 dias, sendo considerado longo. A maior parte dos 
pacientes realizaram procedimentos e tricotomia e banho com clorexidina, porém acreditase que existem falhas no registro.

A maior parte dos pacientes realizou RM, porém a troca de válvula mitral foi mais associada às infecções. As complicações pós-operatórias que apresentaram correlação com infecção foram a FA, IR e IRA. Também houve maior ocorrência de óbito entre os que apresentaram alguma infecção.

O estudo permitiu explorar os principais fatores de risco para ocorrência da IH no pósoperatório de cirurgias cardíacas, além de identificar as principais infecções adquiridas e seus agentes etiológicos. A partir de tais achados propôs-se um protocolo específico para ser utilizado desde a unidade de internação, passando pelo bloco cirúrgico até a UTI, pois se acredita que as medidas preventivas vão muito além daquelas já muitas vezes debatidas no ambiente de cuidados intensivos, estas medidas devem abranger todas as equipes de saúde envolvidas no cuidado ao paciente nos diferentes setores do hospital, devendo existir essa interligação e comunicação eficaz entre eles.

Por conseguinte, a CCIH tem papel primordial na execução das medidas de controle e prevenção de IH, assim como na supervisão das normas e protocolos estabelecidos, porém cada setor do hospital deve assumir o compromisso de trabalhar em conjunto e parceria com tal comissão, no sentido de aprimorar as técnicas e assegurar assistência adequada, principalmente porque a equipe que lida com o paciente à beira do leito é aquela que vai ter a responsabilidade de colocar em prática todas as normas e rotinas orientadas por essa instituição.

Desta forma, acredita-se que o estudo contribui no sentido de estimular tais reflexões e incentivar medidas educativas com base nos achados encontrados, a fim de direcionar esforços para diminuir índices de infecção cada vez mais. Uma das limitações do estudo foi o curto período de tempo para a coleta de dados, além das deficiências de preenchimento encontradas nos prontuários, por se tratar de um estudo retrospectivo. 


\section{REFERÊNCIAS}

ALVES, Thales Gontijo de Siqueira, DAVID, Flégon Lopes, HENRIQUES, Karina Magalhães de Castro, RIANI, Larissa Rodrigues. Prevalência das Principais Complicações Pós-Operatórias em Cirurgias Cardíacas. Revista Brasileira de Cardiologia. v. 24, n. 3, p. 139-146, 2011. Disponível em: < http://sociedades.cardiol.br/socerj/revista/2011_03/a_2011_v24_n03_01prevalencia.pdf >. Acesso em: 02 ago. 2019.

AYRES, M.; AYRES, J. M.; AYRES, D. L.; SANTOS, A. A. S. BioEstat 5.3: Aplicações Estatísticas nas Áreas das Ciências Biológicas e Médicas. 5. ed. Belém-PA: Publicações Avulsas do Mamirauá, 2007.

BECCARIA, Lucia Marinilza; CESARINO, Claudia Bernardi; WERNECK, Alexandre Lins; GÓES, Nadielly Codonho; SANTOS, Karla Soares dos; MACHADO, Maurício de Nassau. Complicações pós-operatórias em pacientes submetidos à cirurgia cardíaca em hospital de ensino. Arq. Ciênc. Saúde. v. 22, n. 1, p. 37-41, 2015. Disponível em: $<$ http://www.cienciasdasaude.famerp.br/index.php/racs/article/view/216>. Acesso em: 14 ago. 2019.

BRASIL, Ministério da Saúde. Conselho Nacional de Saúde. Lei n9431 de janeiro de 1997. Dispõe sobre a obrigatoriedade da manutenção pelos hospitais do país, de Programa de Controle de Infecções Hospitalares. Brasília- DF, $1997 . \quad$ Disponível em: http://bvsms.saude.gov.br/bvs/saudelegis/gm/1998/prt2616_12_05_1998.html>. Acesso em: 14 jun. 2019.

DATASUS. Infarto Agudo do miocárdio é a primeira causa de morte no país, 2014. Disponível em: http://datasus.saude.gov.br/noticias/atualizacoes/559-infarto-agudo-do-miocardio-e-primeira-causa-demortes-no-pais-revela-dados-do-datasus>. Acesso em: 22 mai. 2019.

DUARTE, Elisabeth Carmen; BARRETO, Sandhi Maria. Transição demográfica e epidemiológica: a Epidemiologia e Serviços de Saúde revisita e atualiza o tema. Epidemiol. Serv. Saúde, Brasília-DF. v. 21, n. 4, p. 529-532, 2012. Disponível em: < http://scielo.iec.gov.br/scielo.php?script=sci_arttext\&pid=\$1679-49742012000400001>. Acesso em: 19 mai. 2019.

GERHARDT, Tatiana Engel; SILVEIRA, Denise Toufo. Métodos de pesquisa: organizado por desenvolvimento rural da SEAD/UFRGS- Porto Alegre: Editora da UFRGS, 2009. Disponível em: < http://www.ufrgs.br/cursopgdr/downloadsSerie/derad005.pdf>. Acesso em: 23 jul. 2019.

IZAIAS, Érika Maria; DELLAROZA, Mara Solange Gomes; ROSSANEIS, Mariana Ângela; BELEI, Renata Aparecida. Custo e caracterização de infecção hospitalar em idosos. Ciência \& Saúde Coletiva. Londrina, v. 19, n. 8, p. 33953402, 2014.

KAUFMAN, Renato; KUSCHNIR, Maria Cristina Caetano; XAVIER, Regina Maria Aquino; SANTOS, Marco Aurelio; CHAVES, Rogério Brant Martins; MÜLLER, Regina Elizabeth. Perfil Epidemiológico na Cirurgia de Revascularização Miocárdica. Revista Brasileira Cardiologia. v. 24, n. 6, p. 369-376, 2011.

LEDUR, Priscila; ALMEIDA, Lúcia; PELLANDA, Lucia Campos; SCHAAN, Beatriz D'Agord. Preditores de infecção no pós operatório de cirurgia de revascularização miocárdica. Revista Brasileira de Cirurgia Cardiovascular. v. 26, n. 11, p. 114-127, 2011. Disponível em: < http://www.scielo.br/scielo.php?pid=S010276382011000200008\&script=sci_abstract\&tlng=pt>. Acesso em: 22 jul. 2019.

LIGUORI, Gabriel Romero; KANAS, Alexandre Fligelman; MOREIRA, Luiz Felipe Pinho. Manejo da resposta inflamatória pós circulação extra-corpórea: revisão dos estudos em modelos animais. Rev Bras Cir Cardiovasc. v. 29, n. 1, p. 93-10, 2014. Disponível em: < http://www.scielo.br/scielo.php?script=sci_arttext\&pid=S010276382014000100017>. Acesso em: 07 jun. 2019.

MALTA, Deborah Carvalho; BERNAL, Regina Tomie Ivata; NUNES, Marília Lavocat; OLIVEIRA, Max Moura de; ISER, Betine Pinto Moehlecke; ANDRADE, Silvânia Suely Caribé de Araújo; CLARO, Rafael Moreira; MONTEIRO, Carlos Augusto; SILVA JR, Jarbas Barbosa da. Prevalência de fatores de risco e proteção para doenças crônicas não transmissíveis em adultos: estudo transversal, Brasil, 2011. Epidemiol. Serv. Saúde, Brasília- DF. v. 22, n. 3, 423-434, 2013. Disponível em: < http://www.scielo.br/pdf/ress/v23n4/2237-9622-ress-23-04-00609.pdf>. Acesso em: 09 jun. 2019. 
MENDES, Telma de Almeida Busch; GOLDBAUM, Moisés; SEGRI, Neuber José; BARROS, Marilisa Berti de Azevedo; CESAR, Chester Luiz Galvão; CARANDINA, Luana; ALVES, Maria Cecília Goi Porto. Diabetes mellitus: fatores associados à prevalência em idosos, medidas e práticas de controle e uso dos serviços de saúde em São Paulo, Brasil. Cad. Saúde Pública, Rio de Janeiro. v. 27, n. 6, p. 1233-1243, 2011. Disponível em: < http://www.scielo.br/pdf/csp/v27n6/20.pdf>. Acesso em: 10 jun. 2019.

OLIVEIRA, Adriana Cristina de; KOVNER, Christine Tassone; SILVA, Rafael Souza da. Infecção hospitalar em unidade de tratamento intensivo de um hospital universitário brasileiro. Revista Latino Americana de Enfermagem. v. 18, n.2, p. 97-104, 2010. Disponível em: < http://www.scielo.br/pdf/rlae/v18n2/pt_14.pdf>. Acesso em: 20 jun. 2019.

OLIVEIRA, Júlia de Cássia; FANTINATI, Marcelo Silva. Perfil de pacientes submetidos à cirurgia cardíaca e preditores das complicações pós-operatórias. Revista Biomotriz. v. 7, n. 1, p. 215-24, 2013. Disponível em: <http://www.revistaeletronica.unicruz.edu.br/index.php/BIOMOTRIZ/article/view/215/pdf>. Acesso em: 22 ago. 2019.

PINHEIRO, Raul Henrique Oliveira; VIEIRA, Maria Cristina Umpiérrez; PEREIRA, Evani Marques; BARBOSA, Maria Emilia Marcondes. Fatores de risco para infarto agudo do miocárdio em pacientes idosos cadastrados no programa hiperdia. Cogitare Enferm. v. 18, n. 1, p. 78-83, 2013. Disponível em: < http://bases.bireme.br/cgi-

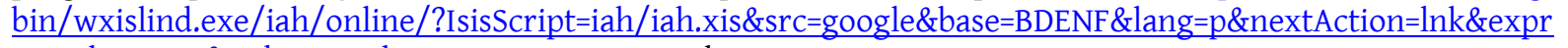
Search=27055\&indexSearch=ID >. Acesso em: 20 jul. 2019.

PRODANOV, Cleber Cristiano; FREITAS, Ernani Cesar de. Metodologia do trabalho científico [recurso eletrônico]: métodos e técnicas da pesquisa e do trabalho acadêmico / Cleber Cristiano Prodanov, Ernani Cesar de Freitas. - 2. ed. - Novo Hamburgo: Feevale, 2013. Disponível em: < http://www.feevale.br/Comum/midias/8807f05a-14d0-4d5b-b1ad-1538f3aef538/E-

book\%20Metodologia\%20do\%20Trabalho\%20Cientifico.pdf>. Acesso em: 17 mai. 2019.

ROCHA, Maiara dos Santos. Estudo das complicações imediatas dos pacientes submetidos à troca valvar mitral com o coração batendo e parado. 37 f. (Monografia) Universidade federal da Bahia, Salvador, 2013.

SAHU, Manoj Kumar; SIDDHARTH, Bharat; CHOUDHURY, Arin; VISHNUBHATLA, Sreenivas; PAL SINGH, Sarvesh; MENON, Ramesh; KAPOOR, Poonam Malhotra; TALWAR, Sachin; CHOUDHARY, Shiv; AIRAN, Balram. Incidence, microbiological profile of nosocomial infections, and their antibiotic resistance patterns in a high volume Cardiac Surgical Intensive Care Unit. Annals of Cardiac Anaesthesia. New Delhi. v. 19, n. 2, p. 281-287, 2016. Disponível em: <https://www.ncbi.nlm.nih.gov/pubmed/27052070>. Acesso em: 22 mai. 2019.

SAMPAIO, Márcio Campos; MÁXIMO, Carlos Alberto Gonçalves; MONTENEGRO, Carolina Moreira; MOTA, Diandro Marinho; FERNANDES, Tatiana Rocha; BIANCO, Antonio Carlos Mugayar; AMODEO, Celso; CORDEIRO, Antonio Carlos. Comparação de critérios diagnósticos de Insuficiência Renal Aguda em cirurgia cardíaca. Arq Bras Cardiol. v. 101, n. 1, p. 18-21, 2013. Disponível em: < http://www.scielo.br/scielo.php?script=sci_arttext\&pid=S0066-782X2013002700005>. Acesso em: 22 jun. 2019.

SANTANA, Rafael Santos; VIANA, Ariane de Carvalho; SANTIAGO, Jozimário da Silva; MENEZES, Michelle Santos; LOBO, Iza Maria Fraga; MARCELLINI, Paulo Sergio. Consequências do uso excessivo de antimicrobianos no pósoperatório: o contexto de um hospital público. Rev. Col. Bras. Cir. Rio de Janeiro. v. 41, n. 3, p. 149-154, 2014. Disponível em: <http://www.scielo.br/pdf/rcbc/v41n3/pt_0100-6991-rcbc-41-03-00149.pdf>. Acesso em: 023 set. 2019.

SANTOS, Augusto Sergio Engenheiro. Pneumonia associada à ventilação mecânica: protocolo de prevenção. Revista UNILUS Ensino e Pesquisa. v. 10, n. 2, p. 52-62, 2013.

SCHMIDTT, Cristiane; LACERDA, Rúbia Aparecida. Fatores relacionados à adesão às recomendações para administração de redose em cirurgias limpas. Rev. Eletr. Enf. v. 17, n. 1, p. 78-84, 2015.

SILVA, Quenia Cristina Gonçalves da; BARBOSA, Maria Helena. Fatores de risco para infecção de sítio cirúrgico em cirurgia cardíaca. Acta Paul Enferm. v. 25, n. 2, p. 89-95, 2012. 
SOARES, Mattos Teixeira Soares; FERREIRA, Diego Costa de Souza, GONÇALVES, Mariana Paula Cunha, SOBRAL, Marcelo Luiz Peixoto; BARBIERI, Lucas Reatieri; GERÔNIMO, Glaucio Mauren da Silva; TROMPIERI, Daniel Francisco; SOUZA, Thierry Araújo de; ROCCA, Juan Marcelo Guilbert; SÁ, Juliano Cavalcante de; TERRAZAS, Anderson da Silva; SANTOS, Gilmar Geraldo dos; STOLF, Noedir Antônio Groppo. O impacto da fibrilação atrial no pós-operatório de revascularização do miocárdio. Relampa. v. 26, n. 3, p. 137-43, 2013. Disponível em: < http://www.relampa.org.br/details/901/pt-BR/o-impacto-da-fibrilacao-atrial-no-posoperatorio-de-revascularizacao-do-miocardio>. Acesso em: 14 jun. 2019.

TEIXEIRA, Diego Codevilla; PEDRO, Fábio Lopes; CARNEIRO, Marcelo. Infecção hospitalar na visão de enfermeiros da Santa Casa de caridade de Bagé - RS. Rev. Epidemiol Control Infec. v. 2, n. 1, p. 14-16, 2012. Disponível em: < https://online.unisc.br/seer/index.php/epidemiologia/article/view/2435>. Acesso em: 10 abr. 2019.

TONIAL, Ricardo; MOREIRA, Daniel Medeiros. Perfil clínico-epidemiológico dos pacientes submetidos à cirurgia de revascularização do miocárdio no instituto de cardiologia de Santa Catarina. Arquivos Catarinenses de Medicina. São José - SC. v. 40, n. 4, p. 0004-2773, 2011.

TORRATI, Fernanda Gaspar; DANTAS, Rosana Ap. Spadoti. Circulação extra-corpórea e complicações no período pós-operatório imediato de cirurgias cardíacas. Acta Paul Enfermagem. v. 25, n. 3, p. 340-5, 2012. Disponível em: < http://www.scielo.br/scielo.php?script=sci_arttext\&pid=S0103-21002012000300004>. Acesso em: 10 jun. 2019 .

WEBSTER, Joan; OSBORNE, Sonya. Preoperative bathing or showeringwith antiseptics to prevent surgical site infection (review). Cochrane Database of Systematic Reviews. Issue. v. 2, n. 4985, 2015.

YI, Qian; LI, Ke; JIAN, Zhao; XIAO, Ying-Bin; CHEN, Lin; ZHANG, Yao; MA, Rui-Yan. Risk factors fo acute kidney injury after cardiovascular surgery: evidence from 2157 cases and 49.777 controls - A meta analysis. Cardiorenal Med. v. 6, n. 3, p. 237-250, 2016. Disponível em: < https://www.ncbi.nlm.nih.gov/pmc/articles/PMC4886037/>. Acesso em: 20 jul. 2019.

(cc) Br

Este trabalho está licenciado com uma Licença Creative Commons - Atribuição 4.0 Internacional. 